\title{
Synthesis of hydroxylated 3,5-diaryl-2-pyrazolines by the reaction of hydroxychalcones with hydrazines
}

\author{
Albert Lévai*a and József Jekő ${ }^{b}$ \\ ${ }^{a}$ Department of Organic Chemistry, University of Debrecen, P.O.Box 20, H-4010 Debrecen, \\ Hungary, ${ }^{b}$ ICN Hungary Co. Ltd., H-4440 Tiszavasvári, Hungary \\ E-mail: alveai@puma.unideb.hu
}

Dedicated to Professor Dr. Alexandru T. Balaban on the occasion of his $75^{\text {th }}$ birthday

(received 27 Apr 05; accepted 22 Jun 05; published on the web 25 Jun 05)

\begin{abstract}
New 1-substituted 3,5-diaryl-2-pyrazolines 13-27 have been synthesized by the reaction of hydroxychalcones 1-12 and hydrazines in hot acetic acid solution. Structures of all new compounds have been elucidated by microanalyses, ${ }^{1} \mathrm{H}$ - and ${ }^{13} \mathrm{C}-\mathrm{NMR}$ spectroscopy.
\end{abstract}

Keywords: Hydroxychalcones, hydrazines, 3,5-diaryl-2-pyrazolines

\section{Introduction}

Pyrazolines are prominent nitrogen-containing heterocyclic compounds and, therefore, various procedures have been worked out for their synthesis. ${ }^{1-4}$ Numerous pyrazoline type compounds have been found to possess useful bioactivity, e.g. antimicrobial, ${ }^{5}$ central nervous system ${ }^{6}$ and immunosuppressive. ${ }^{7}$ Among the various pyrazoline isomers, 2-pyrazolines appear to be the most frequently investigated compounds. As a consequence, a large number of 2-pyrazolines have been described in the chemical literature, using different synthetic methods for their preparation. An especially popular procedure is based on the reaction of $\alpha, \beta$-unsaturated aldehydes and ketones with hydrazines. ${ }^{8-37}$ However, series of specially substituted representatives have been prepared rarely. For this reason, the aim of our present study was to synthesize systematically hydroxylated 3,5-diaryl-2-pyrazolines for the study of their structureactivity relationships. 


\section{Results and Discussion}

Formation of 2-pyrazolines by the reaction of $\alpha, \beta$-unsaturated ketones and hydrazines may take place under various reaction conditions using ethanol, ${ }^{18}$ acetic acid ${ }^{8-10,16,21,25-27}$ or pyridine ${ }^{28,29}$ as solvent. After some preliminary experiments, acetic acid was found to be a convenient solvent in our present case. Hydroxychalcones 1-12 were allowed to react with hydrazine hydrate or phenylhydrazine in hot acetic acid (cf. Experimental Section) to afford 1-substituted 3,5-diaryl-2pyrazolines 13-27 in high yields (Scheme 1). N-Acetylation of all 2-pyrazolines obtained by the reaction of the appropriate chalcones with hydrazine has taken place under these reaction conditions in each case. Formation of the $\mathrm{N}$-acetyl derivatives is beneficial since the 1substituted 2-pyrazolines are stable compounds and can be used for biological and pharmaceutical trials without the risk of undesirable decomposition.

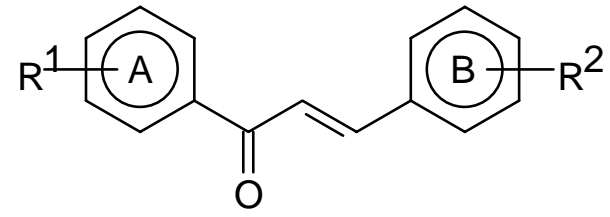

1-12

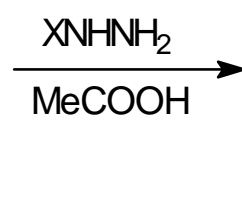

$\mathrm{X}=\mathrm{H}, \mathrm{Ph}$

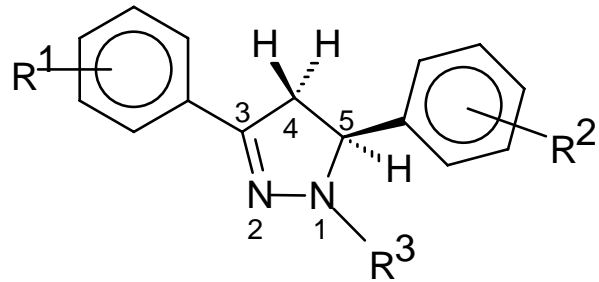

13-27
1, 13: $\mathrm{R}^{1}=2-\mathrm{OH}, \mathrm{R}^{2}=4-\mathrm{iPr}, \mathrm{R}^{3}=\mathrm{AC}$

2, 14: $\mathrm{R}^{1}=2-\mathrm{OH}, \mathrm{R}^{2}=4-\mathrm{Cl}, \mathrm{R}^{3}=A C$

3, 15: $\mathrm{R}^{1}=2-\mathrm{OH}, \mathrm{R}^{2}=2,4-\mathrm{Cl}_{2}, \mathrm{R}^{3}=\mathrm{AC}$

4, 16: $\mathrm{R}^{1}=2-\mathrm{OH}, \mathrm{R}^{2}=2,3-(\mathrm{MeO}) 2, \mathrm{R}^{3}=\mathrm{AC}$

5, 17: $R^{1}=2-\mathrm{OH}, \mathrm{R}^{2}=3,4,5-(\mathrm{MeO})_{3}, \mathrm{R}^{3}=A C$

4, 18: $\mathrm{R}^{1}=2-\mathrm{OH}, \mathrm{R}^{2}=2,3-(\mathrm{MeO})_{2}, \mathrm{R}^{3}=\mathrm{Ph}$

6, 19: $\mathrm{R}^{1}=2-\mathrm{OH}, \mathrm{R}^{2}=2-\mathrm{Cl}, \mathrm{R}^{3}=\mathrm{Ph}$

7, 20: $\mathrm{R}^{1}=2-\mathrm{OH}, \mathrm{R}^{2}=3-\mathrm{Cl}, \mathrm{R}^{3}=\mathrm{Ph}$
8, 21: $R^{1}=3-\mathrm{OH}, \mathrm{R}^{2}=4-\mathrm{MeO}, \mathrm{R}^{3}=A c$

9, 22: $R^{1}=3-\mathrm{OH}, \mathrm{R}^{2}=2,6-\mathrm{Cl}_{2}, \mathrm{R}^{3}=A \mathrm{C}$

9, 23: $\mathrm{R}^{1}=3-\mathrm{OH}, \mathrm{R}^{2}=2,6-\mathrm{Cl}_{2}, \mathrm{R}^{3}=\mathrm{Ph}$

10, 24: $R^{1}=4-\mathrm{OH}, \mathrm{R}^{2}=2,4-\mathrm{Cl}_{2}, \mathrm{R}^{3}=\mathrm{AC}$

11, 25: $R^{1}=4-\mathrm{OH}, \mathrm{R}^{2}=2,6-\mathrm{Cl}_{2}, \mathrm{R}^{3}=A C$

12, 26: $R^{1}=4-\mathrm{OH}, \mathrm{R}^{2}=3,4-\mathrm{Cl}_{2}, \mathrm{R}^{3}=\mathrm{Ac}$

10, 27: $R^{1}=4-\mathrm{OH}, \mathrm{R}^{2}=2,4-\mathrm{Cl}_{2}, \mathrm{R}^{3}=\mathrm{Ph}$

\section{Scheme 1}

Structures of all new compounds 13-27 have been elucidated by elemental analyses, ${ }^{1} \mathrm{H}$ - and ${ }^{13} \mathrm{C}$-NMR measurements. In the ${ }^{1} \mathrm{H}-\mathrm{NMR}$ spectra of 2-pyrazolines 13-27, the three hydrogen atoms attached to the $\mathrm{C}-4$ and $\mathrm{C}-5$ carbon atoms of the heterocyclic ring gave an $\mathrm{ABX}$ spin system. Measured chemical shift and coupling constant values (cf. Experimental Section) unequivocally prove the 2-pyrazoline structure. In the ${ }^{1} \mathrm{H}$-NMR spectra of 1-acetyl-2-pyrazolines 13-17, 21, 22 and 24-36 a singlet signal at around $2.4 \mathrm{ppm}$ refers to the presence of an $N$-acetyl group. In the ${ }^{13} \mathrm{C}-\mathrm{NMR}$ spectra of all new 1-substituted 3,5-diaryl-2-pyrazolines 13-27, the 
chemical shift values of carbon atoms C-3 (154-156 ppm), C-4 (42-44 ppm) and C-5 (56$58 \mathrm{ppm}$ ) corroborate the 2-pyrazoline structure determined by ${ }^{1} \mathrm{H}-\mathrm{NMR}$ spectroscopic measurements. ${ }^{13} \mathrm{C}$-NMR chemical shifts of the $N$-acetyl group also have been assigned in the case of compounds 13-17, 21, 22 and 24-26.

In conclusion, we have synthesized systematically hydroxylated 3,5-diaryl-2-pyrazolines by the reaction of hydrazines and chalcones bearing an ortho-, meta- or para-hydroxy group in their ring $A$. These new substances allow the investigation of the possible linkage of the 2-pyrazolines to various sites in living organisms in the course of the investigation of their bioactivities. The new 1-substituted 3,5-diaryl-2-pyrazolines described in this paper are very stable compounds, a property which may render them especially useful substances in drug research.

\section{Experimental Section}

General Procedures. Melting points were determined with a Koffler hot-stage apparatus and are uncorrected. ${ }^{1} \mathrm{H}$ - and ${ }^{13} \mathrm{C}$-NMR spectra were recorded on a Varian Gemini 200 spectrometer at 200/50 $\mathrm{MHz}$ in $\mathrm{CDCl}_{3}$ (internal standard TMS, $\delta=0.0 \mathrm{ppm}$ ) at room temperature. Elemental analyses were measured in-house with a Carlo Erba 1106 EA instrument. TLC was performed on Kieselgel $60 \mathrm{~F}_{254}$ (Merck) layer using hexane:acetone $(7: 3 \mathrm{v} / \mathrm{v})$ or toluene:ethyl acetate $(4: 1 \mathrm{v} / \mathrm{v})$ as eluents. Starting materials 1-12 were synthesized according to known procedures. ${ }^{38-41}$

\section{General procedure for the synthesis of compounds 13-27}

A mixture of hydroxychalcone (1-12, 10.0 mmoles), hydrazine hydrate $(50.0 \mathrm{mmoles})$ or phenylhydrazine $(50.0$ mmoles $)$ and acetic acid $(40 \mathrm{ml})$ was refluxed for 3 hours then poured into water. The precipitate was separated by filtration, washed free of acid and crystallized from methanol to afford 2-pyrazolines 13-27 (Scheme 1).

1-Acetyl-3-(2-hydroxyphenyl)-5-(4-isopropylphenyl)-2-pyrazoline (13). This compound was obtained as colourless needles in 65\% yield, m.p. $163-164{ }^{\circ} \mathrm{C} ;{ }^{1} \mathrm{H}-\mathrm{NMR}(\delta): 1.22(6 \mathrm{H}, \mathrm{d}, \mathrm{J}=6.9$ $\left.\mathrm{Hz}, \mathrm{CH}\left(\mathrm{CH}_{3}\right)_{2}\right), 2.38\left(3 \mathrm{H}, \mathrm{s}, \mathrm{CH}_{3}\right), 2.89\left(1 \mathrm{H}, \mathrm{m}, \mathrm{CH}\left(\mathrm{CH}_{3}\right)_{2}\right), 3.31(1 \mathrm{H}, \mathrm{dd}, \mathrm{J}=4.6,17.8 \mathrm{~Hz}, 4-$ $\left.\mathrm{H}_{\text {trans }}\right), 3.85\left(1 \mathrm{H}, \mathrm{dd}, \mathrm{J}=11.8,17.8 \mathrm{~Hz}, 4-\mathrm{H}_{\text {cis }}\right), 5.57(1 \mathrm{H}, \mathrm{dd}, \mathrm{J}=4.6,11.8 \mathrm{~Hz}, 5-\mathrm{H}), 6.90-7.41$ $\left(\mathrm{m}, 8\right.$ arom. H), $10.30(1 \mathrm{H}, \mathrm{s}, \mathrm{OH}) ;{ }^{13} \mathrm{C}-\mathrm{NMR}(\delta): 21.9,23.7,33.6,42.6,58.1,115.3,117.2$, $119.8,125.7,127.2,128.6,132.4,138.7,148.8,156.6,157.9,167.9$. Anal. Calcd. for $\mathrm{C}_{20} \mathrm{H}_{22} \mathrm{~N}_{2} \mathrm{O}_{2}$ : C, 74.51; H, 6.88; N, 8.68. Found: C, 74.59; H, 6.84; N, 8.76.

1-Acetyl-5-(4-chlorophenyl)-3-(2-hydroxyphenyl)-2-pyrazoline (14). This material was isolated as colourless needles in 76\% yield, m.p. $146-147{ }^{\circ} \mathrm{C} ;{ }^{1} \mathrm{H}-\mathrm{NMR}(\delta): 2.38\left(3 \mathrm{H}, \mathrm{s}, \mathrm{CH}_{3}\right)$, $3.28\left(1 \mathrm{H}, \mathrm{dd}, \mathrm{J}=4.8,17.9 \mathrm{~Hz}, 4-\mathrm{H}_{\text {trans }}\right), 3.84\left(1 \mathrm{H}, \mathrm{dd}, \mathrm{J}=12.0,17.9 \mathrm{~Hz}, 4-\mathrm{H}_{\text {cis }}\right), 5.54(1 \mathrm{H}, \mathrm{dd}, \mathrm{J}=$ 4.8, $12.0 \mathrm{~Hz}, 5-\mathrm{H}), 6.92-7.41$ (m, 8 arom. H), $10.21(1 \mathrm{H}, \mathrm{s}, \mathrm{OH}) ;{ }^{13} \mathrm{C}-\mathrm{NMR}(\delta): 21.8,42.4,57.7$, 115.0, 117.2, 119.9, 127.2, 128.5, 129.3, 132.5, 133.9, 139.8, 156.3, 157.9, 167.9. Anal. Calcd. for $\mathrm{C}_{17} \mathrm{H}_{15} \mathrm{ClN}_{2} \mathrm{O}_{2}$ : C, 64.87; H, 4.80; N, 8.89. Found: C, 64.98; H, 4.83; N, 8.96. 
1-Acetyl-5-(2,4-dichlorophenyl)-3-(2-hydroxyphenyl)-2-pyrazoline (15). This substance was prepared as pale yellow needles in 71\% yield, m.p. $183-184{ }^{\circ} \mathrm{C} ;{ }^{1} \mathrm{NMR}(\delta): 2.43\left(3 \mathrm{H}, \mathrm{s}, \mathrm{CH}_{3}\right)$, $3.16\left(1 \mathrm{H}, \mathrm{dd}, \mathrm{J}=5.3,18.0 \mathrm{~Hz}, 4-\mathrm{H}_{\text {trans }}\right), 3.92\left(1 \mathrm{H}, \mathrm{dd}, \mathrm{J}=12.0,18.0 \mathrm{~Hz}, 4-\mathrm{H}_{\text {cis }}\right), 5.80(1 \mathrm{H}, \mathrm{dd}, \mathrm{J}=$ 5.3, $12.0 \mathrm{~Hz}, 5-\mathrm{H}), 6.86-7.42$ (m, 7 arom. H), 10.17 (1H, s, OH); ${ }^{13} \mathrm{C}-\mathrm{NMR}(\delta): 21.8,41.6,55.9$, 114.9 , 117.3, 119.9, 127.2, 127.8, 128.6, 130.1, 132.7, 134.4, 136.7, 156.8, 157.9, 168.1. Anal. Calcd. for $\mathrm{C}_{17} \mathrm{H}_{14} \mathrm{Cl}_{2} \mathrm{~N}_{2} \mathrm{O}_{2}$ : C, 58.47; H, 4.04; N, 8.02. Found: C, 58.57; H, 4.07; N, 8.10.

1-Acetyl-5-(2,3-dimethoxyphenyl)-3-(2-hydroxyphenyl)-2-pyrazoline (16). This comp-ound was prepared as colourless plates in $69 \%$ yield, m. p. $166-167{ }^{\circ} \mathrm{C} ;{ }^{1} \mathrm{H}-\mathrm{NMR}(\delta): 2.39(3 \mathrm{H}$, s, $\left.\mathrm{CH}_{3}\right), 3.24\left(1 \mathrm{H}, \mathrm{dd}, \mathrm{J}=5.1,17.8 \mathrm{~Hz}, 4-\mathrm{H}_{\text {trans }}\right), 3.80$ (1H, dd, J = 12.0, $\left.17.8 \mathrm{~Hz}, 4-\mathrm{H}_{\text {cis }}\right), 3.84(3 \mathrm{H}$, $\left.\mathrm{s}, \mathrm{CH}_{3} \mathrm{O}\right), 3.89\left(3 \mathrm{H}, \mathrm{s}, \mathrm{CH}_{3} \mathrm{O}\right), 5.72(1 \mathrm{H}, \mathrm{dd}, \mathrm{J}=5.1,12.0 \mathrm{~Hz}, 5-\mathrm{H}), 6.72-7.37$ (m, 7 arom. $\left.\mathrm{H}\right)$, $10.34(1 \mathrm{H}, \mathrm{s}, \mathrm{OH}) ;{ }^{13} \mathrm{C}-\mathrm{NMR}(\delta): 21.9,42.0,54.5,55.6,60.2,112.1,115.3,116.9,118.6,119.7$, $124.4,128.6,132.2,134.9,146.1,153.1,157.2,157.9$, 167.8. Anal. Calcd. for $\mathrm{C}_{19} \mathrm{H}_{20} \mathrm{~N}_{2} \mathrm{O}_{4}$ : C, 67.04; H, 5.92; N, 8.23. Found: C, 67.15; H, 5.86; N, 8.31.

1-Acetyl-3-(2-hydroxyphenyl)-5-(3,4,5-trimethoxyphenyl)-2-pyrazoline (17). This subs-tance was isolated as colourless needles in $68 \%$ yield, m.p. $154-155{ }^{\circ} \mathrm{C} ;{ }^{1} \mathrm{H}-\mathrm{NMR}(\delta): 2.42(3 \mathrm{H}$, s, $\left.\mathrm{CH}_{3}\right), 3.31\left(1 \mathrm{H}, \mathrm{dd}, \mathrm{J}=5.0,18.0 \mathrm{~Hz}, 4-\mathrm{H}_{\text {trans }}\right), 3.80\left(3 \mathrm{H}, \mathrm{s}, \mathrm{CH}_{3} \mathrm{O}\right), 3.84\left(6 \mathrm{H}, \mathrm{s}, 2 \mathrm{CH}_{3} \mathrm{O}\right), 3.90$ $\left(1 \mathrm{H}, \mathrm{dd}, \mathrm{J}=11.8,18.0 \mathrm{~Hz}, 4-\mathrm{H}_{\mathrm{cis}}\right), 5.49(1 \mathrm{H}, \mathrm{dd}, \mathrm{J}=5.0,11.8 \mathrm{~Hz}, 5-\mathrm{H}), 6.89-7.41$ (m, 6 arom. $\mathrm{H}), 10.28(1 \mathrm{H}, \mathrm{s}, \mathrm{OH}) ;{ }^{13} \mathrm{C}-\mathrm{NMR}(\delta): 21.7,41.2,55.3,57.3,111.7,116.5,119.3,127.1,127.7$, 129.9, 132.5, 134.0, 137.4, 154.2, 160.0, 169.1. Anal. Calcd. for $\mathrm{C}_{20} \mathrm{H}_{22} \mathrm{~N}_{2} \mathrm{O}_{5}$ : C, 64.85; H, 5.99; N, 7.56. Found: C, 64.92; H, 5.93; N, 7.64.

5-(2,3-Dimethoxyphenyl)-3-(2-hydroxyphenyl)-1-phenyl-2-pyrazoline (18). This material was prepared as yellow needles in 70\% yield, mp. $127-128{ }^{\circ} \mathrm{C} ;{ }^{1} \mathrm{H}-\mathrm{NMR}(\delta): 3.23(1 \mathrm{H}, \mathrm{dd}, \mathrm{J}=6.9$, $\left.17.6 \mathrm{~Hz}, 4-\mathrm{H}_{\text {trans }}\right), 3.90\left(3 \mathrm{H}, \mathrm{s}, \mathrm{CH}_{3} \mathrm{O}\right), 3.98\left(3 \mathrm{H}, \mathrm{s}, \mathrm{CH}_{3} \mathrm{O}\right), 4.02(1 \mathrm{H}, \mathrm{dd}, \mathrm{J}=11.3,17.6 \mathrm{~Hz}, 4-$ $\left.\mathrm{H}_{\text {cis }}\right), 5.02(1 \mathrm{H}, \mathrm{dd}, \mathrm{J}=6.9,11.3 \mathrm{~Hz}, 5-\mathrm{H}), 6.82-7.83(\mathrm{~m}, 12$ arom. $\mathrm{H}), 10.89(1 \mathrm{H}, \mathrm{s}, \mathrm{OH}) ;{ }^{13} \mathrm{C}-$ NMR $(\delta): 42.8,55.7,58.1,60.6,111.9,116.6,118.7,119.4,119.8,124.8,127.3,130.4,135.5$, 144.2, 146.0, 150.3, 153.1, 157.4. Anal. Calcd. for $\mathrm{C}_{23} \mathrm{H}_{22} \mathrm{~N}_{2} \mathrm{O}_{3}: \mathrm{C}, 73.78 ; \mathrm{H}, 5.92 ; \mathrm{N}, 7.48$. Found: C, 73.89; H, 5.86; N, 7.57.

5-(2-Chlorophenyl)-3-(2-hydroxyphenyl)-1-phenyl-2-pyrazoline (19). This compound was prepared as pale yellow plates in 66\% yield, m.p. $121-122{ }^{\circ} \mathrm{C} ;{ }^{1} \mathrm{H}-\mathrm{NMR}(\delta): 3.14(1 \mathrm{H}, \mathrm{dd}, \mathrm{J}=$ 7.0, 17.6 Hz, 4- $\left.\mathrm{H}_{\text {trans }}\right), 4.04\left(1 \mathrm{H}, \mathrm{J}=12.1,17.6 \mathrm{~Hz}, 4-\mathrm{H}_{\text {cis }}\right), 5.58(1 \mathrm{H}, \mathrm{dd}, \mathrm{J}=7.0,12.1,5-\mathrm{H})$, 6.80-7.49 (m, 13 arom. H), $10.70(1 \mathrm{H}, \mathrm{s}, \mathrm{OH}) ;{ }^{13} \mathrm{C}-\mathrm{NMR}(\delta): 42.2,60.0,113.2,116.3,116.7$, 119.5, 120.1, 127.4, 127.9, 129.2, 129.4, 130.2, 130.7, 131.9, 138.8, 143.8, 150.2, 157.4. Anal. Calcd. for $\mathrm{C}_{21} \mathrm{H}_{17} \mathrm{ClN}_{2} \mathrm{O}$ : C,n 72.31; H, 4.91; N, 8.03. Found: C, 72.40; H, 4.87; N, 8.09.

5-(3-Chlorophenyl)-3-(2-hydroxyphenyl)-1-phenyl-2-pyrazoline (20). This substance was obtained as yellow plates in $72 \%$ yield, m.p. $122-123{ }^{\circ} \mathrm{C} ;{ }^{1} \mathrm{H}-\mathrm{NMR}(\delta): 3.22(1 \mathrm{H}, \mathrm{dd}, \mathrm{J}=7.6$, $\left.17.3 \mathrm{~Hz}, 4-\mathrm{H}_{\text {trans }}\right), 3.96\left(1 \mathrm{H}, \mathrm{dd}, \mathrm{J}=12.1,17.3 \mathrm{~Hz}, 4-\mathrm{H}_{\text {cis }}\right), 5.20(1 \mathrm{H}, \mathrm{dd}, \mathrm{J}=7.6,12.1 \mathrm{~Hz}, 5-\mathrm{H})$, 6.82-7.40 (m, 13 arom. H), $10.70(1 \mathrm{H}, \mathrm{s}, \mathrm{OH}) ;{ }^{13} \mathrm{C}-\mathrm{NMR}(\delta): 43.8,62.8,113.5,116.2,116.8$, $119.5,120.4,124.2,126.2,127.3,128.3,129.3,130.8,135.3,144.1,149.8,157.5$. Anal. Calcd. for $\mathrm{C}_{21} \mathrm{H}_{17} \mathrm{ClN}_{2} \mathrm{O}$ : C, 72.31, H, 4.91; N, 8.03. Found: 72.22; H, 4.97; N, 7.94. 
1-Acetyl-3-(3-hydroxyphenyl)-5-(4-methoxyphenyl)-2-pyrazoline (21). This material was prepared as colourless plates in 62\% yield, m.p. 211-212 ${ }^{\circ} \mathrm{C} ;{ }^{1} \mathrm{H}-\mathrm{NMR}(\delta): 2.26\left(3 \mathrm{H}, \mathrm{s}, \mathrm{CH}_{3}\right)$, $3.04\left(1 \mathrm{H}, \mathrm{dd}, \mathrm{J}=4.5,18.0 \mathrm{~Hz}, 4-\mathrm{H}_{\text {trans }}\right), 3.70\left(3 \mathrm{H}, \mathrm{s}, \mathrm{CH}_{3} \mathrm{O}\right), 3.80(1 \mathrm{H}, \mathrm{dd}, \mathrm{J}=11.7,18.0 \mathrm{~Hz}, 4-$ $\left.\mathrm{H}_{\text {cis }}\right), 5.47(1 \mathrm{H}, \mathrm{dd}, \mathrm{J}=4.5,11.7 \mathrm{~Hz}, 5-\mathrm{H}), 6.84-7.30$ (m, 8 arom. $\left.\mathrm{H}\right), 10.24(1 \mathrm{H}, \mathrm{s}, \mathrm{OH}) ;{ }^{13} \mathrm{C}-$ NMR $(\delta): 21.6,42.0,55.0,58.8,112.9,117.6,117.8,126.9,129.9,132.6,134.6,154.3,157.8$, 158.7, 167.5. Anal. Calcd. for $\mathrm{C}_{18} \mathrm{H}_{18} \mathrm{~N}_{2} \mathrm{O}_{3}$ : C, 69.66; H, 5.85; N, 9.02. Found: C, 69.78; H, 5.90; N, 9.09 .

1-Acetyl-5-(2,6-dichlorophenyl)-3-(3-hydroxyphenyl)-2-pyrazoline (22). This compound was prepared as yellow needles in 70\% yield, m.p. $252-253{ }^{\circ} \mathrm{C} ;{ }^{1} \mathrm{H}-\mathrm{NMR}(\delta): 2.38\left(3 \mathrm{H}, \mathrm{s}, \mathrm{CH}_{3}\right), 3.27$ $\left(1 \mathrm{H}, \mathrm{dd}, \mathrm{J}=8.5,17.8 \mathrm{~Hz}, 4-\mathrm{H}_{\text {trans }}\right), 3.67\left(1 \mathrm{H}, \mathrm{dd}, \mathrm{J}=12.8,17.8 \mathrm{~Hz}, 4-\mathrm{H}_{\mathrm{cis}}\right), 6.21(1 \mathrm{H}, \mathrm{dd}, \mathrm{J}=8.5$, $12.8 \mathrm{~Hz}, 5-\mathrm{H}), 6.89-7.39$ (m, 7 arom. H), $10.42(1 \mathrm{H}, \mathrm{s}, \mathrm{OH}) ;{ }^{13} \mathrm{C}-\mathrm{NMR}(\delta): 21.2,40.7,56.2$, 112.9, 117.6, 128.7, 129.7, 130.0, 130.2, 132.4, 133.0, 135.1, 135.5, 154.3, 157.8, 167.7. Anal. Calcd. for $\mathrm{C}_{17} \mathrm{H}_{14} \mathrm{Cl}_{2} \mathrm{~N}_{2} \mathrm{O}_{2}$ : C, 58.47; H, 4.04; N, 8.02. Found: C, 58.41; H, 4.01; N, 8.07.

5-(2,6-Dichlorophenyl)-3-(3-hydroxyphenyl)-1-phenyl-2-pyrazoline (23). This substance was obtained as yellow plates in 73\% yield, m.p. $206-207{ }^{\circ} \mathrm{C} ;{ }^{1} \mathrm{H}-\mathrm{NMR}(\delta): 3.31(1 \mathrm{H}, \mathrm{dd}, \mathrm{J}=7.3$, $\left.17.7 \mathrm{~Hz}, 4-\mathrm{H}_{\text {trans }}\right), 3.79\left(1 \mathrm{H}, \mathrm{dd}, \mathrm{J}=11.4,17.7 \mathrm{~Hz}, 4-\mathrm{H}_{\text {cis }}\right), 6.08(1 \mathrm{H}, \mathrm{dd}, \mathrm{J}=7.3,11.4 \mathrm{~Hz}, 5-\mathrm{H})$, 6.76-7.45 (m, 12 arom. H), $10.31(1 \mathrm{H}, \mathrm{s}, \mathrm{OH}) ;{ }^{13} \mathrm{C}-\mathrm{NMR}(\delta): 39.7,60.0,112.3,113.2,115.8$, 118.6, 119.5, 128.6, 129.1, 129.4, 129.9, 130.9, 134.4, 135.2, 135.3, 135.8, 144.6, 146.2, 155.9. Anal. Calcd. for $\mathrm{C}_{21} \mathrm{H}_{16} \mathrm{Cl}_{2} \mathrm{~N}_{2} \mathrm{O}: \mathrm{C}, 65.81 ; \mathrm{H}, 4.21 ; \mathrm{N}, 7.30$. Found: $\mathrm{C}, 65.93 ; \mathrm{H}, 4.27 ; \mathrm{N}, 7.22$.

1-Acetyl-5-(2,4-dichlorophenyl)-3-(4-hydroxyphenyl)-2-pyrazoline (24). This material was obtained as yellow plates in 64\% yield, m.p. $240-241{ }^{\circ} \mathrm{C} ;{ }^{1} \mathrm{H}-\mathrm{NMR}(\delta): 2.31\left(3 \mathrm{H}, \mathrm{s}, \mathrm{CH}_{3}\right), 3.01$ $\left(1 \mathrm{H}, \mathrm{dd}, \mathrm{J}=4.8,18.0 \mathrm{~Hz}, 4-\mathrm{H}_{\text {trans }}\right), 3.88\left(1 \mathrm{H}, \mathrm{dd}, \mathrm{J}=11.8,18.0 \mathrm{~Hz}, 4-\mathrm{H}_{\mathrm{cis}}\right), 5.69(1 \mathrm{H}, \mathrm{dd}, \mathrm{J}=4.8$, $11.8 \mathrm{~Hz}, 5-\mathrm{H}), 6.83-7.66$ (m, 7 arom. H), 10.01 (1H, s, OH); ${ }^{13} \mathrm{C}-\mathrm{NMR}(\delta): 21.4,40.8,56.6$, 115.7, 121.9, 127.9, 128.0, 128.6, 129.3, 131.9, 132.7, 138.5, 154.6, 159.9, 167.5. Anal. Calcd. for $\mathrm{C}_{17} \mathrm{H}_{14} \mathrm{Cl}_{2} \mathrm{~N}_{2} \mathrm{O}_{2}$ : C, 58.47; H, 4.04; N, 8.02. Found: C, 58.53; H, 4.07; N, 8.09.

1-Acetyl-5-(2,6-dichlorophenyl)-3-(4-hydroxyphenyl)-2-pyrazoline (25). This compound was prepared as pale yellow needles in 70\% yield, m.p. $261-262{ }^{\circ} \mathrm{C} ;{ }^{1} \mathrm{H}-\mathrm{NMR}(\delta): 2.20\left(3 \mathrm{H}, \mathrm{s}, \mathrm{CH}_{3}\right)$, $3.15\left(1 \mathrm{H}, \mathrm{dd}, \mathrm{J}=7.9,18.0 \mathrm{~Hz}, 4-\mathrm{H}_{\text {trans }}\right), 3.80\left(1 \mathrm{H}, \mathrm{dd}, \mathrm{J}=12.9,18.0 \mathrm{~Hz}, 4-\mathrm{H}_{\text {cis }}\right), 6.06(1 \mathrm{H}, \mathrm{dd}, \mathrm{J}=$ 7.9, $12.9 \mathrm{~Hz}, 5-\mathrm{H}), 6.84-7.65$ (m, 7 arom. H), 9.98 (1H, s, OH); ${ }^{13} \mathrm{C}-\mathrm{NMR}(\delta): 21.2,40.7,56.0$, $115.7,122.1,128.5,128.8,129.7,130.2$, 133.0, 135.1, 135.7, 154.3, 159.8, 167.4. Anal. Calcd. for $\mathrm{C}_{17} \mathrm{H}_{14} \mathrm{Cl}_{2} \mathrm{~N}_{2} \mathrm{O}_{2}$ : C, 58.47; H, 4.04; N, 8.02. Found: C, 58.39; H, 3.99; N, 8.08.

1-Acetyl-5-(3,4-dichlorophenyl)-3-(4-hydroxyphenyl)-2-pyrazoline (26). This substance was isolated as yellow plates in $67 \%$ yield, m.p. $254-255{ }^{\circ} \mathrm{C} ;{ }^{1} \mathrm{H}-\mathrm{NMR}(\delta): 2.28\left(3 \mathrm{H}, \mathrm{s}, \mathrm{CH}_{3}\right), 3.12$ $\left(1 \mathrm{H}, \mathrm{dd}, \mathrm{J}=4.9,18.1 \mathrm{~Hz}, 4-\mathrm{H}_{\text {trans }}\right), 3.79\left(1 \mathrm{H}, \mathrm{dd}, \mathrm{J}=11.7,18.1 \mathrm{~Hz}, 4-\mathrm{H}_{\mathrm{cis}}\right), 5.51(1 \mathrm{H}, \mathrm{dd}, \mathrm{J}=4.9$, $11.7 \mathrm{~Hz}, 5-\mathrm{H}), 6.83-7.64\left(\mathrm{~m}, 7\right.$ arom. H), $10.01(1 \mathrm{H}, \mathrm{s}, \mathrm{OH}) ;{ }^{13} \mathrm{C}-\mathrm{NMR}(\delta): 21.5,41.8,58.2$, 115.7, 122.0, 126.1, 128.1, 128.7, 129.9, 131.1, 143.7, 154.6, 159.9, 167.6. Anal. Calcd. for $\mathrm{C}_{17} \mathrm{H}_{14} \mathrm{Cl}_{2} \mathrm{~N}_{2} \mathrm{O}_{2}$ : C, 58.47; H, 4.04, N, 8.02. Found: C, 58.56; H, 4.07; N, 8.09.

5-(2,4-Dichlorophenyl)-3-(4-hydroxyphenyl)-1-phenyl-2-pyrazoline (27). This substance was prepared as yellow plates in 83\% yield, m.p. 125-126 ${ }^{\circ} \mathrm{C} ;{ }^{1} \mathrm{H}-\mathrm{NMR}(\delta): 3.01(1 \mathrm{H}, \mathrm{dd}, \mathrm{J}=6.9$, $\left.17.2 \mathrm{~Hz}, 4-\mathrm{H}_{\text {trans }}\right), 3.94\left(1 \mathrm{H}, \mathrm{dd}, \mathrm{J}=11.8,17.2 \mathrm{~Hz}, 4-\mathrm{H}_{\text {cis }}\right), 5.51(1 \mathrm{H}, \mathrm{dd}, \mathrm{J}=6.9,11.8 \mathrm{~Hz}, 5-\mathrm{H})$, 
6.75-7.62 (m, 12 arom. H), $10.30(1 \mathrm{H}, \mathrm{s}, \mathrm{OH}) ;{ }^{13} \mathrm{C}-\mathrm{NMR}(\delta): 41.9,60.8,113.2,115.7,119.4$, $125.5,127.7,128.1,128.6,129.2,129.8,132.6,134.0,138.2$, 144.8, 147.4, 156.6. Anal. Calcd. for $\mathrm{C}_{21} \mathrm{H}_{16} \mathrm{Cl}_{2} \mathrm{~N}_{2} \mathrm{O}: \mathrm{C}, 65.81 ; \mathrm{H}, 4.21 ; \mathrm{N}, 7.30$. Found: $\mathrm{C}, 65.72 ; \mathrm{H}, 4.17 ; \mathrm{N}, 7.38$.

\section{Acknowledgements}

The present study was sponsored by the Hungarian National Research Foundation (Grant No. OTKA T049468) for which our gratitude is expressed. Technical assistance of Mrs. M. Nagy is highly appreciated.

\section{References}

1. Wiley, K. H., Ed. Pyrazoles, Pyrazolines, Pyrazolidines, Indazoles and Condensed Rings, In The Chemistry of Heterocyclic Compounds, Weissberger, A., Ed.; Interscience Publishers: New York, 1967; Vol. 22, p 180.

2. Elguero, J. In Comprehensive Heterocyclic Chemistry II, Katritzky, A. R.; Rees, C.W.; Scriven, E. F.V., Eds; Pergamon Press: Oxford, 1996, Vol.3, p1.

3. Lévai, A. Khim. Geterotsikl. Soedin. 1997, 747.

4. Lévai, A. J. Heterocyclic Chem. 2002, 39,1.

5. Ramalingham, K.; Thyvekikakath, G. X.; Berlin, K. D.; Chesnut, R. W.; Brown, R. A.; Durham, N. N.; Ealick, A. E.; van der Helm, D. J. Med. Chem. 1977, $20,847$.

6. Brown, R. E.; Shavrel Jr., J. US Patent 1972, 3,624,102; Chem. Abstr. 1972, 76, 59618.

7. Lombardino, J. G.; Otterness, I. G. J. Med. Chem. 1981, 24, 830.

8. Raiford, L. C.; Peterson, W. J. J. Org. Chem. 1936, 1, 544.

9. Raiford, L. C.; Gundy, G. V. J. Org. Chem. 1938, 3, 265.

10. Raiford, L. C.; Manley, R. H. J. Org. Chem. 1940, 5, 590.

11. Ried, W.; Dankert, G. Chem. Ber. 1957, 90, 2707.

12. Wiley, R. H.; Jarboe, C. H.; Hayes, F. N.; Hansbury, E.; Nielsen, J. T. ; Callahan, P. X.; Sellars, M. C. J. Org. Chem. 1958, 23, 732.

13. Sammour, A. E. A. Tetrahedron 1964, 20, 1067.

14. Bhatnagar, I; George, M. V. Tetrahedron 1968, 24, 1293.

15. Aubagnac, J. L; Elguero, J.; Jacquier, R. Bull. Soc. Chim. Fr. 1969, 3292.

16. Weber, F. G.; Brosche, K.; Seedorf, C.; Rinow, A. Monatsh. Chem. 1969, 100, 1924.

17. Joshi, M. G; Wadodkar, K. N. Indian J. Chem. 1981, 20B, 1090.

18. Sharma, T. C.; Pawar, S. R; Reddy, N. J. Acta Chim. Hung. 1983, 112, 159.

19. Dhar, D. N.; Raghunathan, R. Indian J. Chem. 1984, 23B, 1187.

20. Orlov, V. D.; Aziz, M. A.; Mchedlov-Petrosyan, N. O.; Asoka, P. K. D. Khim. Geterotsikl. Soedin. 1985, 1511. 
21. Sachchar, S. P.; Singh, A. K. J. Indian Chem. Soc. 1985, 62, 142.

22. Lévai, A.; Szöllősy, Á; Tóth, G. J. Chem. Research (S) 1985, 392.

23. Tóth, G.; Szöllősy, Á.; Lóránd, T.; Szabó, D.; Földesi, A.; Lévai, A. J. Chem. Soc., Perkin Trans. 2 1989, 319.

24. Szöllősy, Á.; Tóth, G.; Lóránd, T.; Kónya, T.; Aradi, F.; Lévai, A. J. Cherm. Soc., Perkin Trans. 2 1991, 489.

25. Andorta, C. S.; Khajuria, J.; Singh, G. B.; Singh, S. J. Indian Chem. Soc. 1993, 70, 266.

26. Bilgin, A. A.; Palaska, E.; Sunal, R.; Gümüsel, B. Pharmazie 1994, 49, 67.

27. Mishriky, N.; Asaad, F. M.; Ibrahim, Y. A.; Girgis, A. S. Pharmazie 1996, 51, 544.

28. Lévai, A. J. Heterocyclic Chem. 1998, 35, 13.

29. Lévai, A. Heterocycl. Commun. 1999, 5, 151.

30. Dighade, S. R.; Chincholcar, M. M. Asian J. Chem. 2001, 13, 1606.

31. Wang, P.; Onozawa-Komatsuzaki, N.; Himeda, Y.; Sugihara, H.; Arakawa, H.; Kasuga K. Tetrahedron Lett. 2001, 42, 9199.

32. Manna, F.; Chimenti, F.; Bolasco, A.; Secci, D.; Bizzarri, B.; Befani, O.; Turini, P.; Mondovi, B.; Alcaro, S.; Tafi, A. Bioorg. Med. Chem. Lett. 2002, 12, 3629.

33. Lévai, A.; Patonay, T.; Silva, A. M. S.; Pinto, D. C. G. A.; Cavaleiro, J. A. S. J. Heterocyclic Chem. 2002, 39, 751.

34. Lévai, A. Heterocycl. Commun. 2003, 9, 287.

35. Lévai, A.; Silva, A. M. S.; Pinto, D. C. G. A.; Cavaleiro, J. A. S.; Alkorta, I.; Elguero, J.; Jekö, J. Eur. J. Org. Chem. 2004, 4672.

36. Chimenti, F.; Bolasco, A.; Manna, F.; Secci, D.; Chimenti, P.; Befani, O.; Turini, P.; Giovannini, V.; Mondovi, B.; Cirilli, R.; La Torre, F. J. Med. Chem. 2004, 47, 2071.

37. Chimenti, F.; Bizzarri, B.; Manna, F.; Boalsco, A.; Secci, D.; Chimenti, P.; Granese, A.; Rivanera, D.; Lilli, D.; Scaltrito, M. M.; Brenciaglia, M. I. Mioorg. Med. Chem. Lett. 2005, $15,603$.

38. Jha, B. C.; Amin, G. C. Tetrahedron 1958, 2, 241.

39. Cavaleiro, J. A. S.; Elguero, J.; Jimeno, L.; Silva, A. M. S. Chem. Lett. 1991, 445.

40. Silva, A. M. S.; Price, W. A.; Cavaleiro, J. A. S. Tetrahedron Lett. 1993, 34, 5657.

41. Stoyanov, E. V.; Champavier, Y.; Simon, A.; Basly, J. P. Bioorg. Med. Chem. Lett. 2002, 12, 2685. 